\title{
A Simple and Cost-Effective Weight Drop Model to Induce Contusive Spinal Cord Injury: Functional and Histological Outcomes
}

\author{
Shiva Hashemizadeh ${ }^{1}$, Saereh Hosseindoost ${ }^{2}$, Khalil Pestei ${ }^{3}$ and Mahmoudreza Hadjighassem ${ }^{1,2, *}$ \\ ${ }^{1}$ Department of Neuroscience and Addiction Studies, School of Advanced Technologies in Medicine, Tehran University of Medical Sciences, Tehran, Iran \\ ${ }^{2}$ Brain and Spinal Cord Injury Research Centre, Neuroscience Institute, Tehran University of Medical Sciences (TUMS), Tehran, Iran \\ ${ }^{3}$ Department of Anesthesiology, Imam Khomeini Hospital Complex, Tehran University of Medical Sciences, Tehran, Iran \\ Corresponding author: Brain and Spinal Cord Injury Research Centre, Neuroscience Institute, Tehran University of Medical Sciences (TUMS), Tehran, Iran. Email: \\ mhadjighassem@tums.ac.ir \\ Received 2021 August 17; Accepted 2021 October 12.
}

\begin{abstract}
Background: Animal spinal cord injury (SCI) models have provided a better perception of the mechanisms related to traumatic SCI and evaluation of the effectiveness of experimental therapeutic interventions.

Objectives: The aim of this study is to develop a cost-effective modified Allen's device to induce contusive spinal cord injury. Methods: Adult male Wistar rats were subjected to contusive spinal cord injury using a customized weight drop model through 10-g weights delivered from a $25-\mathrm{mm}$ height onto an exposed spinal cord. Locomotor and sensory function during 28 days were assessed. Moreover, histopathological changes were assessed at one week and 28 days post SCI.

Results: All the SCI rats showed hind limb paralysis up to $48 \mathrm{~h}$ post SCI and neuropathic pain after injury. Histological changes similar to the previous reports for contusion model were observed.

Conclusions: According to our findings, little variability was observed in the BBB score of individual rats at 28 days after injury. Our customized device to induce spinal cord injury is a simple and inexpensive alternative method to the highly sophisticated contusion device commonly used to induce SCI.
\end{abstract}

Keywords: Spinal Cord Injury, Animal Model, Rats, Contusion

\section{Background}

Traumatic spinal cord injury (SCI) is generally considered as a sudden damage to the spinal cord from an external mechanical force. The primary injury damages cells and initiates secondary cascades of cellular, biochemical, and vascular events that exacerbate neural function (1). It can also lead to permanent deficits in the spinal structure and functions and even cause death. Spinal cord injury is characterized by sensory, motor, and autonomic dysfunction at or below the level of injury. Spinal cord injury represents a major public health problem and remains the most frequent life-long disability that significantly impairs a patient's quality of life (2).

Currently, the most common causes of SCI in the developing countries are falls, motor vehicle accidents, and sport-related injuries. The global incidence of SCI in the developing countries has been estimated at 2.1 to 130.7 per million (3). But in under-developed countries such as Iran, based on the latest update, the prevalence of SCI is an estimated 318.45 per million (4). In this regard, any attempt to understand the pathophysiology of SCI and develop new treatment strategies is critical. Therefore, pre-clinical experiments must be designed with an aim to provide better insight into the secondary mechanism and potential therapy to address the problem $(5,6)$. Because of the limitation of the clinical setting, animal models are essential for studying the cellular and molecular events of human SCI.

The SCI model was selected based on the aim of research. Contusion, compression, transection, and chemical models are the most widely used animal models to induce SCI (7-9). Indeed, both contusion and compression models are most frequently used to better stimulate human secondary mechanism and are more common in clinical patients (10). However, because of the complexity of human SCI, animal models are not able to mimic all aspects of the human pathology of injury. Currently, rodent models are widely used due to their size, low cost, ease of handling, and fast reproduction (11). Rats are widely applied because of their close pathological relationship to humans, involving proven similarities, including formation of cavities and fluid-filled cysts (12). It is obvious now 
that the contusion model has contributed to a much better understanding of the cellular and molecular mechanisms of SCI and its subsequent neuropathological outcomes. Initially, the Allen weight drop model was developed in 1911 to deliver the impact to the intact dura through a laminectomy that leads to controlled spinal cord contusion, which was first employed on a dog. Later on, modified models were developed to induce injury in rodents to promote understanding of the pathophysiology of SCI.

Three types of contusion devices commonly used to induce SCI in rats include New York University's MASCIS impactor (NYU), Ohio State University's electromagnetic spinal cord injury device (OSU impactor), and the Infinite horizon $(\mathrm{IH})$ devices $(1,13)$. Each of these devices has its own advantage and limitation. Although these instruments came into use considerably, the use of these sophisticated instruments is not available in all research labs. In addition, several factors limit the use of these tools, such as high cost, need for highly trained operators, and sophisticated software (1).

\section{Objectives}

The purpose of this study was to develop a costeffective, simple, and feasible device to produce experimental contusion spinal cord injuries.

\section{Methods}

\subsection{Animals}

A total of 20 adult male Wistar rats (240 - $260 \mathrm{~g}$ ) were used. The rats were fed standard laboratory food, provided tap water ad libitum, and kept in a conditioned animal room $\left(23^{\circ} \mathrm{C}, 12\right.$-hour artificial light-dark cycle). All animal studies were approved by the Institutional Animal Care and Use Committee at Tehran University of Medical Sciences. For animal modeling purposes, the animals were separated into the two groups of contusion injured $(n=13)$ and control group $(n=7)$. All animals of the injured group received a spinal contusion, as described below. Animals of the control group did not undergo any surgical interventions.

\subsection{Customized Weight Drop Instrument to Create Contusive Spinal Cord Injury}

In this study, we designed a customized and costeffective weight drop instrument based on the weight drop Allen model (14) with some modifications to induce contusive spinal cord injury. The image of the apparatus and its different components (Borj Sanat Co., Tehran, Iran) are presented in Figure 1. The device is composed of four major parts: (1) guiding metal tube with holes drilled all along at regular intervals to induce graded injury; (2) stainless metal impounder placed symmetrically to the center of the exposed spinal cord with a $2.5-\mathrm{mm}$ diameter. It is positioned at the end of the metal tube, which has contact with the exposed spinal cord and prevents it from bouncing after impact; (3) a metal rod of $10 \mathrm{~g}$ weight with a $2.5 \mathrm{~mm}$ diameter, which is supported by the pin and is dropped from $25 \mathrm{~mm}$ height onto the exposed spinal cord; (4) a pair of fine artery forceps that is positioned on the mobile base plate. It is necessary to stabilize the vertebral column in the horizontal situation by holding the dorsal spine process rostral and caudal to the laminectomy site in order to avoid asymmetrical injury; (5) the most important part of this device is controlling the dropped weight, which is related to a wire which does not allow the weight fully suppress the spinal cord and can be called the controlled contusive weight drop model.

\subsection{Surgical Procedure}

Under sterile surgical conditions, the rats were anesthetized using a mixture of ketamine ( $80 \mathrm{mg} / \mathrm{kg}$ ) and xylazine $(10 \mathrm{mg} / \mathrm{kg})$. Sterile eye drops were used to moisten eyes during anesthesia. After removal of hair, the surgical area was cleaned with betadine. Then, a midline dorsal incision (approximately $2.5 \mathrm{~cm}$ ) was done on the rats. Dorsal laminectomy at T10/T11 vertebrates was performed first by removing the vertebral spinous process using a micro rongeur. We applied a minimally invasive method and only removed the T10 or T11 lamina to achieve an efficient model. Then, the dorsal lamina of only one segment (T10 vertebra) was drilled carefully with motorized dental handpiece in order to expose the spinal cord (Figures $2 \mathrm{~A}$ $\mathrm{B})$. Then, the rat was placed on an impactor device, and the vertebral column was fixed with two forceps rostral and caudal to the laminectomy site (Figures $2 \mathrm{C}$ - E). Finally, the metal rod was released, which impacted the spinal cord (Figure 2F). The spinal cord injury model was verified by the observation of the hind-limbs twitching and picking up the tail of the animal. Next, the injured muscles were sutured, and the skin was closed with suture. The rats showed hind-limb paralysis immediately after thoracic injury (Figure $2 G$ ).

\subsection{Post-surgical Care}

Two rats were housed in a cage after the surgery. In addition to postoperative care, the animals were subcutaneously administrated $1 \mathrm{~mL}$ of $0.9 \%$ saline and $5 \mathrm{mg} / \mathrm{kg}$ of gentamicin intraperitoneally once a day until five days following the operation to prevent urinary tract infection. In 

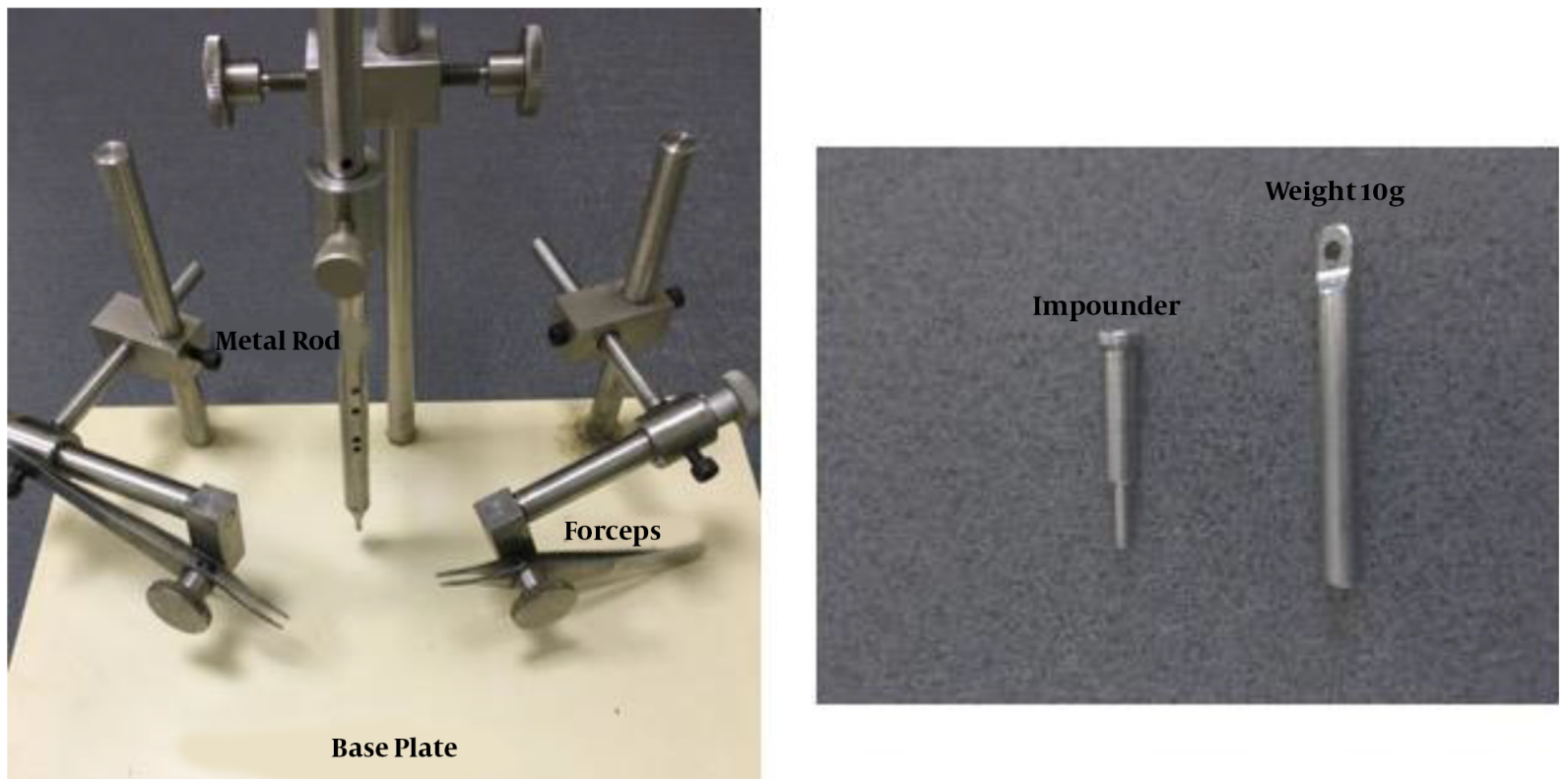

Figure 1. Photograph of the modified weight drop model used for thoracic contusive spinal cord injury model

all the rats, the bladder was manually expressed twice a day until spontaneous bladder function returned.

\subsection{Behavioral Test for the Validation of Contusive Spinal Cord:} Locomotor Function and Thermal Hyperalgesia

\subsubsection{Open Field Locomotion Testing}

Hind-limb motor function of all the rats was assessed using the open field Basso-Beattie-Bresnahan (BBB) rating scale as described previously by (15). Briefly, BBB locomotor rating scale is a grading score ranging from zero (fully paralyzed) to 21 points (complete functional recovery). BassoBeattie-Bresnahan represents locomotor activity according to the joint's movement and forelimb and hind-limb coordination, which indicate functional recovery after SCI. Behavioral alterations were assessed before surgery, on days one and three post-injury, and subsequently, once per week for 28 days after SCI. Scores for the left and right limbs were averaged.

\subsubsection{Hot-Plate}

The hot-plate test (Borj Sanat, Tehran) was used to evaluate thermal nociception. Briefly, all groups of rats were habituated to the room temperature test $1 \mathrm{~h}$ before the test. During the tests, the rats were placed on a metal surface at a constant temperature of $52 \pm 0.5^{\circ} \mathrm{C}$. Response latency, which is the time taken to observe a nociceptive behavior from the plate (foot-licking, jumping, or rapidly removing paw or vocalization), was recorded on day o (before the surgery), 7, 14, 21, and 28 after the SCI induction. The rats were removed from the hot-plate surface once a reaction was observed or after a 20-s cut-off time if no response was observed to prevent tissue damage at $52^{\circ} \mathrm{C}$. Each animal was tested twice separated with a 30 -minute interval between them (16).

\subsection{Histology}

To measure the lesion area and moto neuron survival, H\&E and toluidine blue were measured. Four rats were randomly selected in SCI group and sacrificed at 1 weeks and 4 weeks after SCI for histopathological examination. All rats were deeply anesthetized with ketamine and perfused transcardially first with physiological saline, followed by $100 \mathrm{~mL} 4 \%$ paraformaldehyde in $0.1 \mathrm{M}$ phosphatebuffered saline (PBS), pH 7.4 to pre-fix. The lesion center of spinal cord was dissected and post-fixed in formalin overnight. Sections were embedded in paraffin and cut into $9 \mu \mathrm{m}$ thick transverse sections. Spinal cord sections were stained with hematoxylin \& eosin ( $\mathrm{H} \& \mathrm{E}$ ) or toluidine blue using the standard protocol. Five toluidine blue stained sections were randomly selected to calculate the average numbers of ventral motor neurons. The sections were visualized under an optic microscope. Five sections from each animal were randomly selected for neuronal counts. 

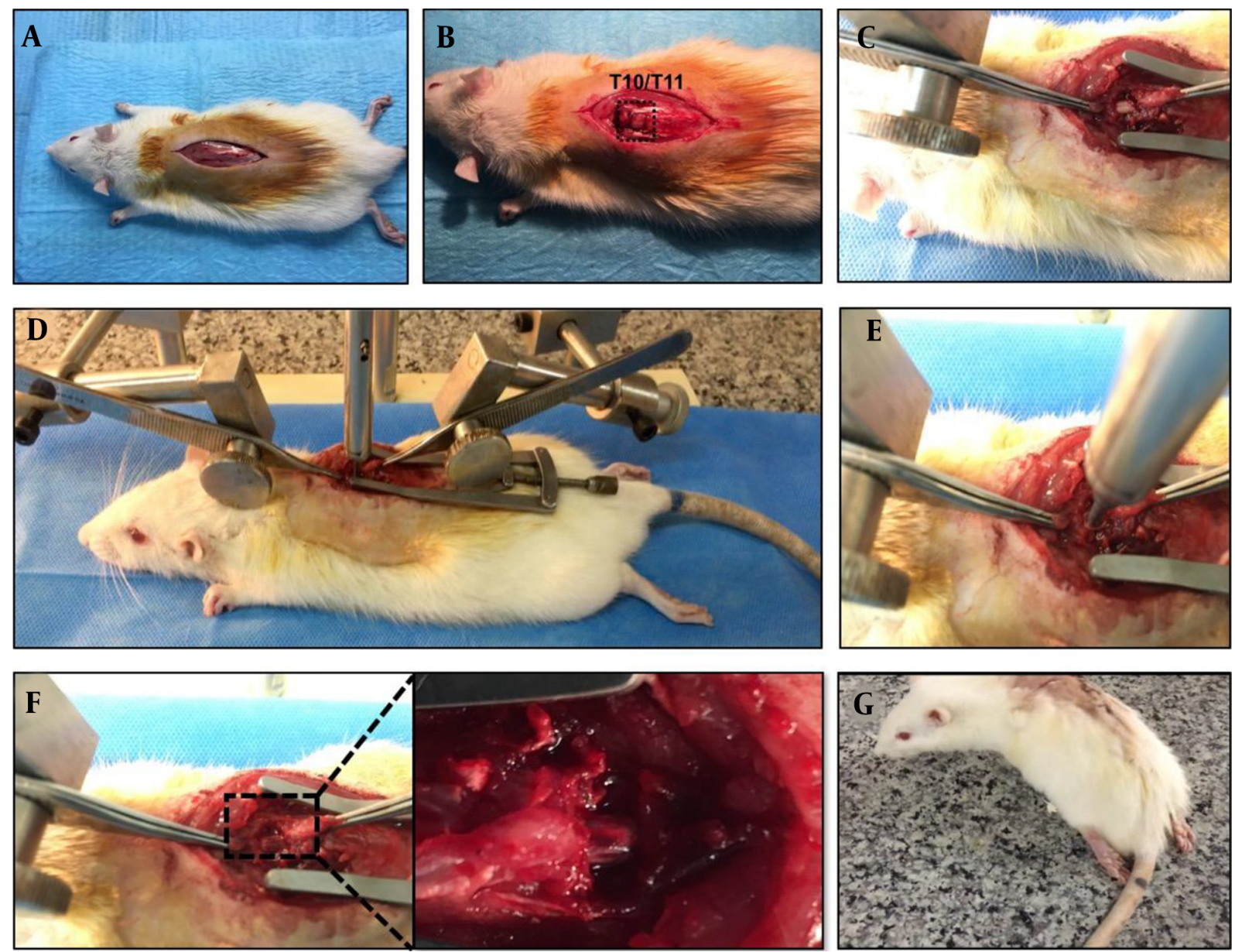

Figure 2. Spinal cord thoracic contusion model; (A) Anesthetize rat under ketamine and xylazine then hair was removed. A longitudinal midline dorsal incision was made after being sterilized with betadine; B-C, The T10-T11 lamina was removed to expose the spinal cord; D, The rat was placed under the device, and the spinal column was stabilized with forceps; E- F, Impact injury was performed by releasing the removable pin; G, Hind limp paralysis one day post injury was noted.

\section{Results}

All the animals in SCI group tolerated the procedure well. The assessment of locomotion by BBB and sensory test by hot-plate was carried out during 4 weeks after surgery. Animals were sacrificed 7 and 28 days post SCI, and spinal cord was dissected out and sent for histopathological outcome.

\subsection{Locomotor and sensory}

We developed a cost-effective weight drop model to induce contusive thoracic spinal cord injury. The behavioral alternation in the SCI group was examined during four weeks. A sensitive and reliable BBB scoring system was used to estimate the severity and recovery of locomotor function following the thoracic SCI. Prior to the SCI, all the animals showed a normal BBB score. Based on the
BBB scores, the animals were measured for two consecutive days after surgery, and no limb movement was observed, revealing complete hind-limb paralysis (Figure $2 G$ ). The average BBB score was 2 at 4 days after SCI. Slight spontaneous improvement was observed in SCI animals, and BBB score reached a plateau at 28 days. The mean BBB score at 28 days was about 9 , indicating plantar step. However, no hind limb-fore limb coordination suggests no recovery was observed in the SCI group at the end of four weeks (Figure $3 \mathrm{~A}$ ) and $\mathrm{BBB}$ score reached a functional plateau on day 21.

To evaluate sensory function at different time points after SCI, we estimated thermal hyperalgesia using the hotplate test (Figure 3B). Paw withdrawal latency in response to thermal stimulation in rats from control and SCI groups was measured. Thresholds of hind-paw withdrawal were constant during four weeks in the control group (7 - $8 \mathrm{~s}$ ). 
One week after injury, the animals showed a significant decrease in thermal response, which persisted throughout the experiment during four weeks in SCI rats $(\mathrm{P}<0.001$ for all the groups compared to the control).

\subsection{Histological Change}

We also examined the histological changes of the spinal cord at lesion site at one and four weeks post-injury. The $\mathrm{H} \& \mathrm{E}$ stained sections from the injury model indicated that a large central cavity at lesion segment, which is characteristic of contusive injuries in rats. Our results indicated tissue loss after spinal cord injury at one and four weeks post SCI (Figure 3C). Toluidine blue staining was done to evaluate the numbers of motor neurons in the spinal cord anterior horn the spinal cord one and four weeks after SCI. Toluidine blue staining revealed a higher decrease in the number of motor neurons in the anterior horn of the injured spinal cord of rats than in that of the control rats (Figure 3D).

\section{Discussion}

Animal models of human SCI are valuable tools to understanding the secondary mechanism of the injury to achieve new therapeutic findings. Based on the study design, appropriate animal models should be selected or modified (17). For SCI research, the most important point is to establish an ideal animal model similar to clinical SCI. Furthermore, technical availability, low cost, feasibility, and reproducibility of the device to stimulate injury are important to develop an ideal animal model. We described the SCI model using a modified weight drop device to excite a specific height force by the impounder for inducing controlled contusive injury. We selected a $25-\mathrm{mm}$ fall height, which was applied in the NYU impactor weight drop model to induce moderate injury in the rats. By using an impounder in our device, the risk of multiple injuries was reduced due to bouncing of a weight when dropped to the spinal cord. Therefore, this design leads to an accurate, precise, and specific model compared to the conventional weight loss method and the NYU shock.

Based on recent findings, in this experiment, we applied novel surgical techniques instead of conventional methods to expose the spinal cord, including laminectomy, using the motorized drill (18). We also applied a minimally invasive technique (19) to remove only the T10 or T11 vertebra segment to achieve an efficient spinal cord model.

Several behavioral paradigms have been used to measure the neurological impairment following SCI, including motor test, sensory test, locomotor test, and sensory motor test (7). Behavioral assessment should also be selected on the basis of reliability, validation, and sensitivity. BBB score and thermal sensory are commonly used in spinal cord contusion in rats. We obtained a mean BBB score of 9 for the 25-mm height injury. Consistent with previous studies, motor deficits are observed following spinal cord injury using a similar weight drop model or other experimental SCI models, such as NYU and Infinite horizon models of spinal cord injury. Motor deficits produced with our device are consistent with the BBB scores obtained from other weight drop devices, such as NYU and IH devices.

A previous study investigated thoracic contusive injury results in hind-paw heat hyper-reactivity after SCI(20). Our results showed that response to the thermal stimulus in the SCI group was faster than the sham group in our designed model.

In addition, the severity of neurological deficits from mild to moderate injury could be increased by changing the dropping height in our device. We showed that this device uses a simple procedure to create repeatable spinal cord injury with less variability among SCI rats. The behavioral and histological findings in the presented study indicated that this device can be used to establish moderate to severe SCI models. Based on the findings of this study, our designed SCI model can be a valid and suitable model for inducing spinal cord injury in rats in experimental SCI studies.

\section{Acknowledgments}

We are grateful to the Brain and Spinal Cord Injury Research Centre, Neuroscience Institute, Tehran University of Medical Sciences, Tehran, Iran.

\section{Footnotes}

Authors' Contribution: S.H. conceived, designed, and drafted the manuscript and analyzed and interpreted the data; S.H. participated in drafting of the manuscript; K.P. revised the manuscript; M.H. performed the critical revision of the manuscript for important intellectual content and study supervision.

Conflict of Interests: The authors declare no conflicts of interest.

Data Reproducibility: The data presented in this study are available on request from the corresponding author.

Ethical Approval: All animal studies were approved by the Institutional Animal Care and Use Committee at Tehran University of Medical Sciences.

Funding/Support: This study was financially supported by the Brain and Spinal Cord Injury Research Centre, neuroscience institute, TUMS. 
A

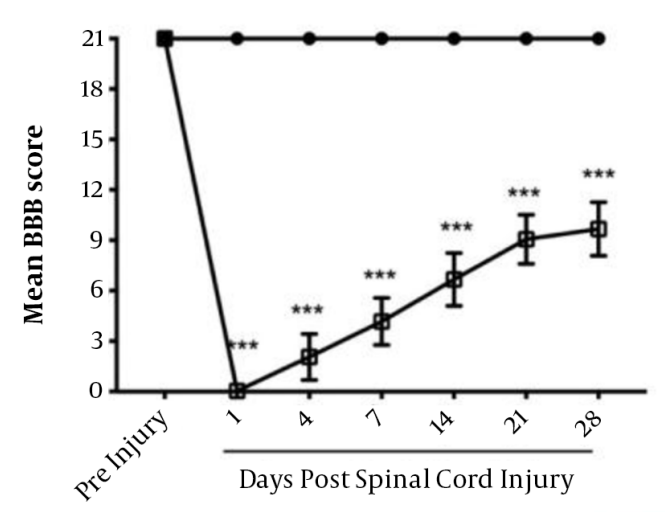

B Hot-Plate

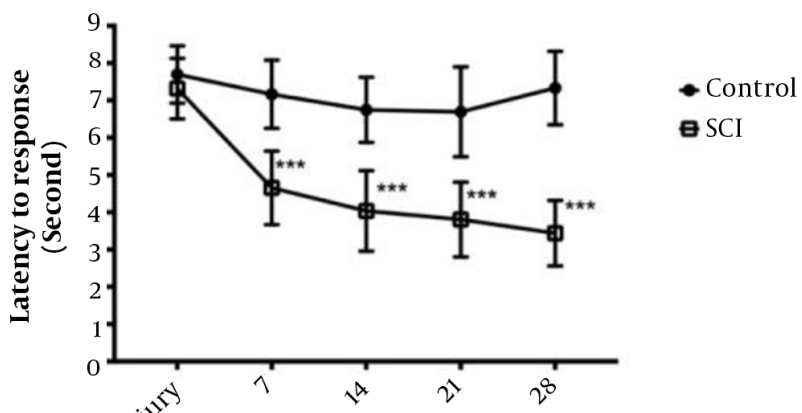

Days Post Spinal Cord Injury

C
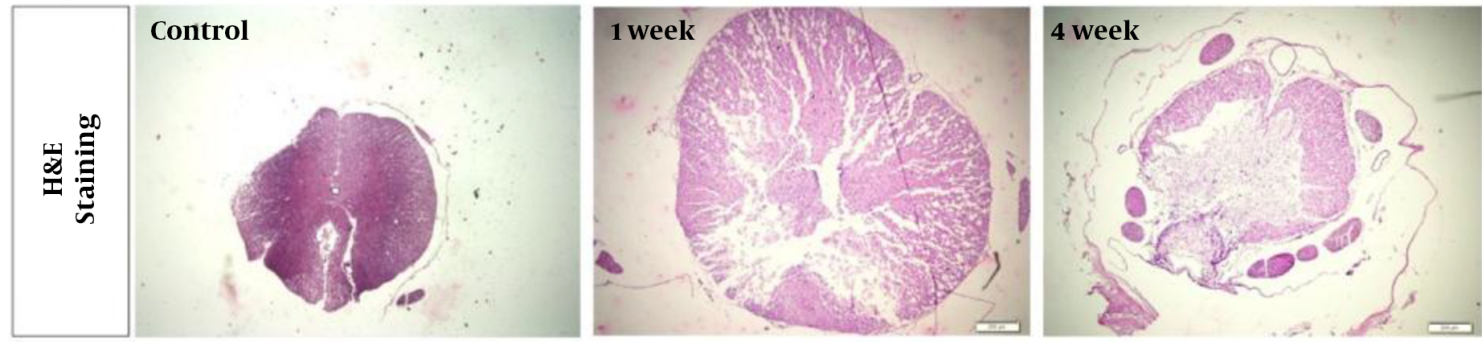

D

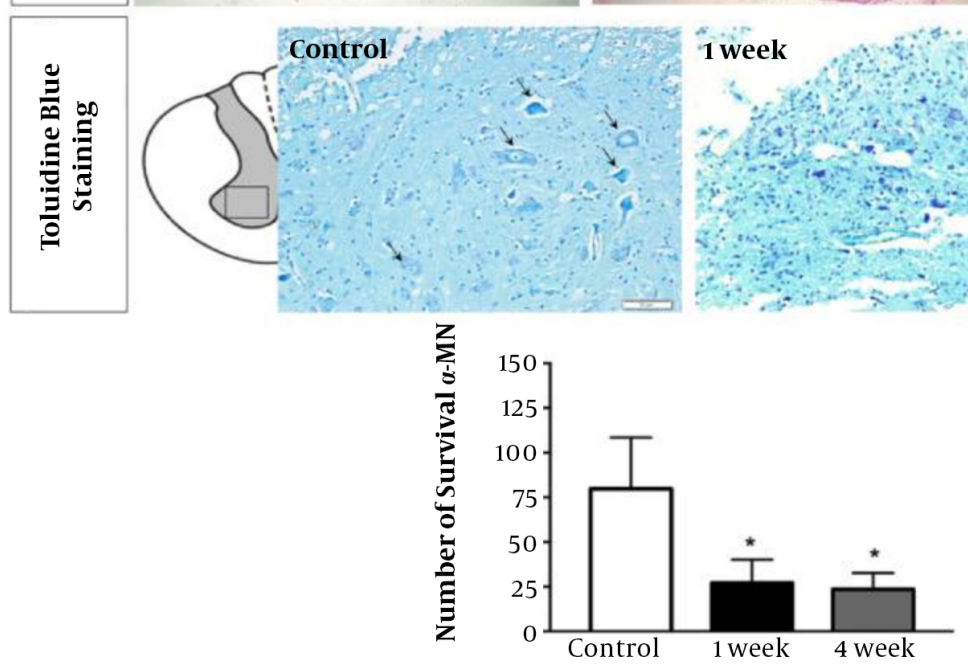

Figure 3. A, BBB score test showing hind-limb partial recovery until four weeks after SCI. The scores differed between the sham and spinal cord injury groups 28 days post spinal cord injury; B, Thermal hyperalgesia by the hot-plate test in rats subjected to SCI. The scores differed between the sham and spinal cord injury groups 28 post spinal cord injury [*** $\mathrm{P}<0.001$; $t$-test revealed a significant difference in latency to response in the SCI group $(\mathrm{n}=13)$ compared to the sham group $(\mathrm{n}=7)$ ]. $\mathrm{C}$ - $\mathrm{D}$, Lesion center of the rat spinal cord C, H \& E image; and D, toluidine blue staining of injured spinal cord at one and four weeks post-SCI. Changes to the spinal cord structure at one week showed little changes, whereas at week 4 cavitation and loss of tissue mass were noted.

\section{References}

1. Alizadeh A, Dyck SM, Karimi-Abdolrezaee S. Traumatic Spinal Cord Injury: An Overview of Pathophysiology, Models and Acute Injury Mechanisms. Front Neurol.2019;10:282. doi: 10.3389/fneur.2019.00282. [PubMed: 30967837]. [PubMed Central: PMC6439316].

2. Priebe MM, Chiodo AE, Scelza WM, Kirshblum SC, Wuermser LA, Ho $\mathrm{CH}$. Spinal cord injury medicine. 6. Economic and societal issues in spinal cord injury. Arch Phys Med Rehabil. 2007;88(3 Suppl1):S84-8. doi: 10.1016/j.apmr.2006.12.005. [PubMed: 17321854].
3. Rahimi-Movaghar V, Sayyah MK, Akbari H, Khorramirouz R, Rasouli MR, Moradi-Lakeh M, et al. Epidemiology of traumatic spinal cord injury in developing countries: a systematic review. Neuroepidemiology. 2013;41(2):65-85. doi: 10.1159/000350710. [PubMed: 23774577].

4. Jazayeri SB, Ataeepour M, Rabiee H, Motevalian SA, Saadat S, Vaccaro AR, et al. Prevalence of Spinal Cord Injury in Iran: A 3-Source Capture-Recapture Study. Neuroepidemiology. 2015;45(1):28-33. doi: 10.1159/000435785. [PubMed: 26201388]. 
5. Cizkova D, Murgoci AN, Cubinkova V, Humenik F, Mojzisova Z, Maloveska M, et al. Spinal Cord Injury: Animal Models, Imaging Tools and the Treatment Strategies. Neurochem Res. 2020;45(1):134-43. doi: 10.1007/s11064-019-02800-w. [PubMed: 31006093].

6. Abdullahi D, Annuar AA, Mohamad M, Aziz I, Sanusi J. Experimental spinal cord trauma: a review of mechanically induced spinal cord injury in rat models. Rev Neurosci.2017;28(1):15-20. doi:10.1515/revneuro2016-0050. [PubMed: 27845888].

7. Ahmed RU, Alam M, Zheng YP. Experimental spinal cord injury and behavioral tests in laboratory rats. Heliyon. 2019;5(3). e01324. doi: 10.1016/j.heliyon.2019.e01324. [PubMed:30906898]. [PubMed Central: PMC6411514].

8. Ahuja CS, Wilson JR, Nori S, Kotter MRN, Druschel C, Curt A, et al. Traumatic spinal cord injury. Nat Rev Dis Primers. 2017;3:17018. doi: 10.1038/nrdp.2017.18. [PubMed: 28447605].

9. Cheriyan T, Ryan DJ, Weinreb JH, Cheriyan J, Paul JC, Lafage V, et al. Spinal cord injury models: a review. Spinal Cord. 2014;52(8):588-95. doi: 10.1038/sc.2014.91. [PubMed: 24912546].

10. Verma R, Virdi JK, Singh N, Jaggi AS. Animals models of spinal cord contusion injury. Korean J Pain. 2019;32(1):12-21. doi: 10.3344/kjp.2019.32.1.12. [PubMed: 30671199]. [PubMed Central: PMC6333579].

11. Kjell J, Olson L. Rat models of spinal cord injury: from pathology to potential therapies. Dis Model Mech. 2016;9(10):1125-37. doi: 10.1242/dmm.025833. [PubMed: 27736748]. [PubMed Central: PMC5087825].

12. Nardone R, Florea C, Holler Y, Brigo F, Versace V, Lochner P, et al. Rodent, large animal and non-human primate models of spinal cord injury. Zoology (Jena). 2017;123:101-14. doi: 10.1016/j.zool.2017.06.004. [PubMed: 28720322].

13. Park JH, Kim JH, Oh SK, Baek SR, Min J, Kim YW, et al. Analysis of equivalent parameters of two spinal cord injury devices: the New York University impactor versus the Infinite Horizon impactor. Spine J. 2016;16(11):1392-403. doi: 10.1016/j.spinee.2016.06.018. [PubMed: 27349631].

14. Allen AR. Surgery of Experimental Lesion of Spinal Cord Equivalent to Crush Injury of Fracture Dislocation of Spinal Column. JAMA. 1911;57(11):878-80. doi:10.1001/jama.1911.04260090100008.

15. Basso DM, Beattie MS, Bresnahan JC. A sensitive and reliable locomotor rating scale for open field testing in rats. $J$ Neurotrauma. 1995;12(1):1-21. doi: 10.1089/neu.1995.12.1. [PubMed: 7783230].

16. Dale CS, Pagano Rde L, Paccola CC, Pinotti-Guirao T, Juliano MA, Juliano L, et al. Effect of the C-terminus of murine S100A9 protein on experimental nociception. Peptides. 2006;27(11):2794-802. doi: 10.1016/j.peptides.2006.07.002. [PubMed:16920226].

17. Sharif-Alhoseini M, Khormali M, Rezaei M, Safdarian M, Hajighadery A, Khalatbari MM, et al. Animal models of spinal cord injury: a systematic review. Spinal Cord. 2017;55(8):714-21. doi: 10.1038/sc.2016.187. [PubMed: 28117332].

18. Harikrishnan VS, Palekkodan H, Fasaludeen A, Krishnan LK, Abelson KSP. Refinement of the spinal cord injury rat model and validation of its applicability as a model for memory loss and chronic pain. Heliyon. 2021;7(7). e07500. doi: 10.1016/j.heliyon.2021.e07500. [PubMed: 34286145]. [PubMed Central: PMC8278431].

19. Duan H, Pang Y, Zhao C, Zhou T, Sun C, Hou M, et al. A novel, minimally invasive technique to establish the animal model of spinal cord injury. Ann Transl Med. 2021;9(10):881. doi: 10.21037/atm-21-2063. [PubMed: 34164515]. [PubMed Central: PMC8184457].

20. Duan W, Huang Q, Chen Z, Raja SN, Yang F, Guan Y. Comparisons of motor and sensory abnormalities after lumbar and thoracic contusion spinal cord injury in male rats. Neurosci Lett. 2019;708:134358. doi: 10.1016/j.neulet.2019.134358. [PubMed: 31269465]. 